\title{
miRNA-195 suppresses cell proliferation of ovarian cancer cell by regulating VEGFR2 and AKT signaling pathways
}

\author{
JUN CHEN

\begin{abstract}
Department of The Third Gynecological, The Third Affiliated Hospital of Qiqihar Medical College, Qiqihar, Heilongjiang 161000, P.R. China
\end{abstract}

Received June 14, 2017; Accepted January 31, 2018

DOI: $10.3892 / \mathrm{mmr} .2018 .9098$

\begin{abstract}
The present study aimed to investigate the functional effects of microRNA-195 on ovarian cancer cells and the underling mechanism involved. Reverse transcription-quantitative polymerase chain reaction was used to measure the expression of microRNA-195 in patients with ovarian cancer. Cell proliferation and apoptosis were measured with MTT assay and flow cytometry, respectively. Caspase-3/9 activity, vascular endothelial growth factor receptor (VEGFR)2 and phosphorylated protein kinase B (p-AKT) protein expression were analyzed using caspase-3/9 activity kits and western blot analysis. The expression of microRNA-195 was downregulated in ovarian cancer, compared with the normal control group. Furthermore, microRNA-195 suppresses cell proliferation and induced apoptosis of ovarian cancer cells. In addition, microRNA-195 suppressed VEGFR2 and p-AKT protein expression in ovarian cancer cells. The inhibition of VEGFR2 and p-AKT increased the functional effects of microRNA-195 on apoptosis of ovarian cancer cells. The results demonstrated that microRNA-195 suppresses cell proliferation of ovarian cancer cells through regulation of VEGFR2 and AKT signaling pathways.
\end{abstract}

\section{Introduction}

Morbidity of ovarian cancer ranks the third among malignancies of the female genital tract. However, ovarian cancer ranks first regarding mortality (1). Ovarian cancer has indistinct clinical symptoms and hidden onset, and there is currently no reliable early detection method (1). Thus, $\sim 70 \%$ of ovarian cancer patients are in advanced stages at initial diagnosis. In addition, patients have frequently developed extensive metastases. It is estimated that the 5-year survival rate among

Correspondence to: Dr Jun Chen, Department of The Third Gynecological, The Third Affiliated Hospital of Qiqihar Medical College, 27 Taishun Road, Qiqihar, Heilongjiang 161000, P.R. China E-mail: jclo767022@163.com

Key words: microRNA-195, ovarian cancer, vascular endothelial growth factor receptor 2, protein kinase B these patients is only $30-45 \%$ (2). Epithelial ovarian cancer (EOC) accounts for $80-90 \%$ of all ovarian cancer cases. It is the most common pathological type of ovarian cancer. The standard treatment regimen for patients with EOC at first diagnosis is maximum cytoreductive surgery with postoperative adjuvant chemotherapy, most commonly platinum-based chemotherapy (2).

A feature of microRNA (miR) expression is its tissue specificity (3). Research has demonstrated that miR expression abundance and function are different in tumor tissues of various histological origins (4). miR expression in various types of tumor tissues and normal control tissues have been analyzed (5). The results suggest that tumors of different histological types have distinct miR expression profiles (5). Furthermore, different tumor types can be distinguished through the differentially expressed miRs. Currently, research on miRs generally concentrates on investigating tumorigenesis, development and tumor-targeted therapy (6). miRs have an important regulatory role in the genesis and development of human tumors. This suggests that miRs may become a novel direction for the diagnosis and treatment of human tumor (4). Zhang et al (7) demonstrated that serum miR-195 is a novel non-invasive biomarker for the detection of cervical cancer.

Vascular endothelial growth factor (VEGF) specifically binds the vascular endothelial growth factor receptor (VEGFR) (8). Thus, it has a vital role in tumor invasion and metastasis (3). Recent research has demonstrated that ovarian cancer cells express VEGFR and VEGF. This indicates that VEGF can directly act on ovarian cancer cells in an autocrine and paracrine manner (9). Previous studies have verified that VEGF is involved in the genesis, development, invasion and metastasis of ovarian cancer (10).

Protein kinase B (AKT) is a serine/threonine protein kinase. It is also the downstream target protein of phosphoinositide 3-kinase (PI3K) (10). In addition, it is also the central link of the PI3K/AKT signal transduction pathway. AKT is involved in the genesis and development of multiple tumors (11). AKT regulates cell survival, apoptosis, proliferation, malignant tumorigenesis and chemoresistance (12). The expression of AKT has been demonstrated to be upregulated in ovarian cancer. In addition, AKT activation can inhibit ovarian cancer cell apoptosis (11) and is involved in the genesis, development, invasion and metastasis of ovarian cancer (13). Sun et al (14) indicate that miR-195 suppressed cell growth in renal cell 
carcinoma via PI3K/AKT signaling pathways. Therefore, the present study aimed to investigate the functional effects of miR-195 on ovarian cancer cells and the underlying mechanism involved.

\section{Materials and methods}

Clinical cohorts. Peripheral blood samples from female patients with ovarian cancer $(n=8)$ and healthy female volunteers $(n=8)$ were collected and these patients were diagnosed at different stages at the Department of Third Gynecological, Third Affiliated Hospital of Qiqihar Medical College (Qiqihar, China) between December 2015 and March 2016. Patient details are presented in Table I. A total of $5 \mathrm{ml}$ peripheral blood was obtained from patients with ovarian cancer and healthy volunteers and following centrifugation at 2,000 x g for $10 \mathrm{~min}$ at $4^{\circ} \mathrm{C}$, the obtained serum samples were preserved at $-80^{\circ} \mathrm{C}$.

Cell culture and transfections. Human OVCAR-3 ovarian cancer cells were purchased from Type Culture Collection of the Chinese Academy of Sciences (Shanghai, China) and cultured in RPMI-1640 medium (Invitrogen; Thermo Fisher Scientific, Inc., Waltham, MA, USA) supplemented with $10 \%$ fetal bovine serum (FBS; Invitrogen; Thermo Fisher Scientific, Inc.) at $37^{\circ} \mathrm{C}$ in a humidified incubator with $5 \%$ $\mathrm{CO}_{2}$. miR-195 (5'-UAGCAGCACAGAAAUGGC-3'), miR-195 inhibitor (5'-CAGUACUUUUGUGUAGUACAA-3') and negative mimic (5'-CAGUACUUUUGUGUAGUACAA-3') were purchased from Guangzhou RiboBio Co., Ltd. (Guangzhou, China). OVCAR-3 cells were transfected with $100 \mathrm{nM}$ miR-195, miR-195 inhibitor and negative mimic using Lipofectamine $2000^{\circledR}$ (Invitrogen; Thermo Fisher Scientific, Inc.) according to the manufacturer's protocol. After transfection for $4 \mathrm{~h}$, AKT inhibitor (100 nM; LY294002) or VEGFR2 inhibitor (10 nM; vandetanib) was added post-transfection for $48 \mathrm{~h}$ at $37^{\circ} \mathrm{C}$. The control group was transfected with negative mimic.

Reverse transcription-quantitative polymerase chain reaction (RT-qPCR) and microarray samples. Total RNA was extracted from cell or serum sample using a TRIzol ${ }^{\mathrm{TM}}$ reagent (Invitrogen; Thermo Fisher Scientific, Inc.). Total RNA (200 ng) was converted into cDNA using the RevertAid First Strand cDNA Synthesis kit (Thermo Fisher Scientific, Inc.). qPCR was accomplished using FastStart Universal SYBR Green Master mix (Rox; Invitrogen; Thermo Fisher Scientific, Inc.) by the ABI PRISM ${ }^{\circledR} 7300$ real-time PCR system (Applied Biosystems; Thermo Fisher Scientific, Inc.). The thermocycling conditions were as follows: $94^{\circ} \mathrm{C}$ for $3 \mathrm{~min} ; 40$ cycles of $94^{\circ} \mathrm{C}$ for $30 \mathrm{sec}, 65^{\circ} \mathrm{C}$ for $40 \mathrm{sec}$ and $72^{\circ} \mathrm{C}$ for $30 \mathrm{sec}$; final extension at $72^{\circ} \mathrm{C}$ for $5 \mathrm{~min}$. The primer sequences were as follows: miR-195, forward 5'-ACACTCCAGCTGGGTAGCAGCACA GAAAT-3' and reverse 5'-TGGTGTCGTGGAGTCG-3'; U6, forward 5'-CTCGCTTCGGCAGCACA-3' and reverse 5'-AAC GCTTCACGAATTTGCGT-3'. Relative expression level was computed using $2^{-\Delta \Delta \mathrm{Cq}}$ method (15).

Total RNA (200 ng) was amplified by Low Input Quick-Amp Labeling kit (Agilent Technologies, Inc., Santa Clara, CA, USA) and labeled with Cy3 (Agilent Technologies, Inc.). Cy3-labeled cRNAs were used for hybridization in
Table I. Demographic and clinical features of patients with ovarian cancer and healthy volunteers.

\begin{tabular}{lcc}
\hline Characteristics & $\begin{array}{c}\text { Ovarian cancer } \\
(\mathrm{n}=8)\end{array}$ & $\begin{array}{c}\text { Healthy volunteers } \\
(\mathrm{n}=8)\end{array}$ \\
\hline Age (years) & $48.92 \pm 8.35$ & $47.44 \pm 7.34$ \\
TNM stage & & \\
I & 2 \\
II & 5 \\
III-IV & \\
Type & 6 \\
Carcinoma & 2 \\
Adenocarcinoma & \\
\hline
\end{tabular}

TNM, tumor, node and metastasis staging.

TaqMan ${ }^{\circledR}$ Array Standard Plates (cat. no. 4413266, Thermo Fisher Scientific, Inc.). Cy3-signal scanned images were quantified using a Feature Extraction 10.5.1.1 image analysis software (Agilent Technologies, Inc.).

Cell proliferation assay. Following transfection for 24, 48 and $72 \mathrm{~h}$, cell viability was measured by the MTT assay. A total of $20 \mu \mathrm{l}$ MTT $(0.5 \mathrm{mg} / \mathrm{ml}$; Sigma-Aldrich; Merck KGaA, Darmstadt, Germany) was added into each well and incubated for $4 \mathrm{~h}$ at $37^{\circ} \mathrm{C}$. Then, the medium was removed and $150 \mu \mathrm{l}$ dimethyl sulfoxide (Sigma-Aldrich; Merck KGaA) were added to cell and incubated for $20 \mathrm{~min}$ at $37^{\circ} \mathrm{C}$. The optical density was detected with a Multiskan EX (Thermo Fisher Scientific, Inc.) at $490 \mathrm{~nm}$.

Apoptosis rate. Following transfection for 48 h, OVCAR-3 cells were harvested and stained with Annexin V-PE and propidium iodide using an Apoptosis kit (BD Pharmingen; BD Biosciences, Franklin Lakes, NJ, USA) according to the manufacturer's protocol. The rate of apoptosis was quantified with a flow cytometer (FACSCalibur ${ }^{\mathrm{TM}}$ system; BD Biosciences) and apoptosis rate was analyzed using FlowJo software (version 7.6.1; FlowJo LLC, Ashland, OR, USA).

Caspase-3/9 activity. Caspase-3/9 activity of cells was measured using Caspase-3/9 activity kits (Beyotime Institute of Biotechnology, Shanghai, China) following transfection for 48 h. The optical density was detected with a Multiskan EX (Thermo Fisher Scientific, Inc.) at $450 \mathrm{~nm}$.

Western blotting. Following transfection for $48 \mathrm{~h}$, cell proteins were extracted using radioimmunoprecipitation assay buffer (Beyotime Institute of Biotechnology) and the protein concentration was determined using a bicinchoninic acid kit (Beyotime Institute of Biotechnology). Equal amount of proteins $(30-50 \mu \mathrm{g})$ were separated on 8-12\% SDS-PAGE and transferred to a polyvinylidene difluoride membrane (Mai Bio Co., Ltd., Shanghai, China). The membrane was blocked using 5\% skimmed milk for $1 \mathrm{~h}$ at $37^{\circ} \mathrm{C}$ and incubated with $\mathrm{Bcl} 2$ associated $\mathrm{X}$ apoptosis regulator (Bax; 1:1,000; cat no. sc-6236; Santa Cruz Biotechnology), VEGFR2 (1:2,000; 
A

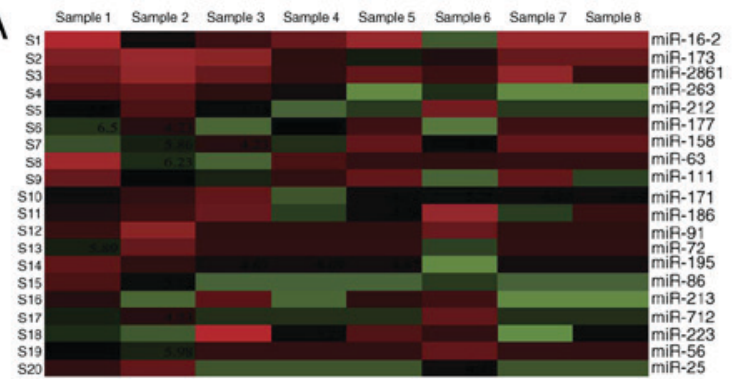

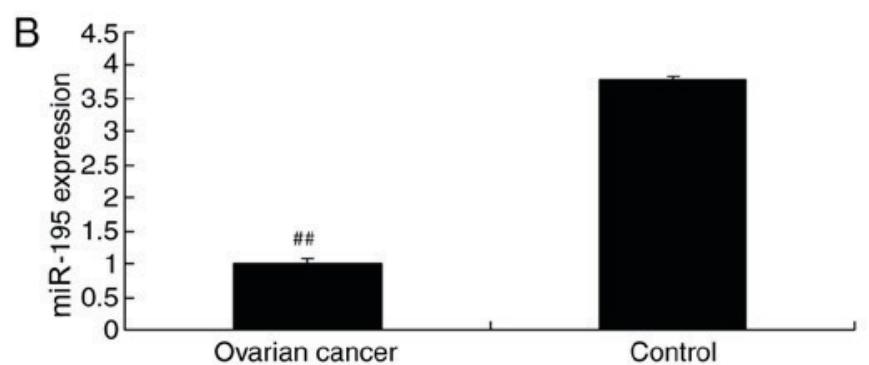

Figure 1. Expression of miR-195 in ovarian cancer. The expression of miR-195 in serum samples was determined using (A) microarray chip and (B) reverse transcription-quantitative polymerase chain reaction. Healthy volunteers were used as the control group. Samples 1-4 are patients with ovarian cancer, samples 5-8 are healthy controls. ${ }^{* \#} \mathrm{P}<0.01$ vs. control group. miR, microRNA.
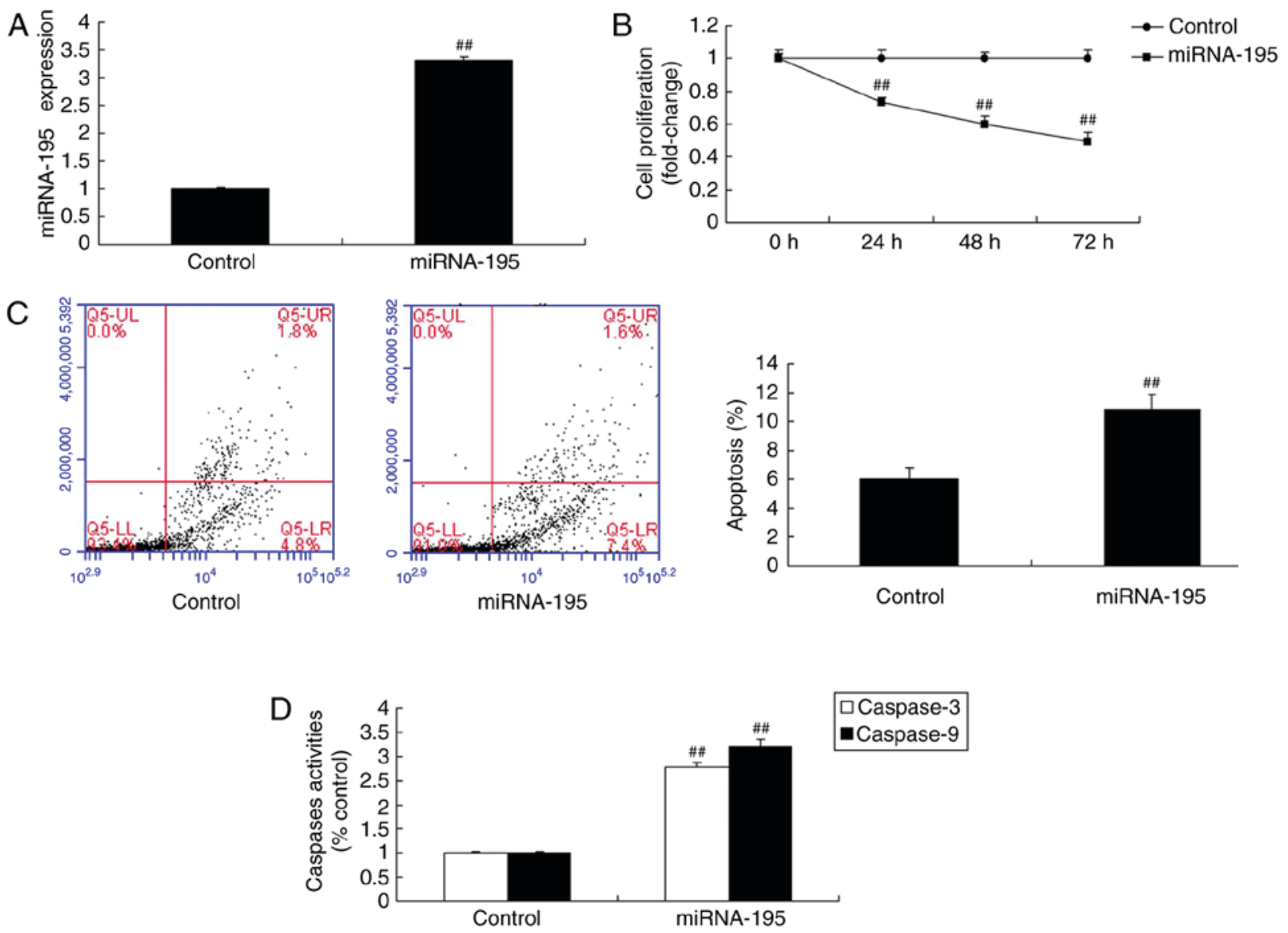

Figure 2. miRNA-195 suppresses cell proliferation and induced apoptosis of ovarian cancer cell. (A) Expression of miRNA-195, (B) cell proliferation and (C) apoptosis rate. Q5-UR and Q5-LR were designated as apoptotic cells. (D) Caspase-3/9 activity. ${ }^{\# \#<0.01 ~ v s . ~ t h e ~ c o n t r o l ~ g r o u p . ~ C o n t r o l, ~ n e g a t i v e ~ m i m i c ~}$ control group; miRNA-195, microRNA-195; Q, quadrant; UR, upper right; UL, upper left; LR, lower right; LL, lower left.

sc-6236; Santa Cruz Biotechnology), phosphorylated (p)-AKT (1:1,000; cat no. sc-7985-R; Santa Cruz Biotechnology) and GAPDH (1:5,000; cat no. ab8245; Abcam, Cambridge, UK) at $4^{\circ} \mathrm{C}$ overnight. The membranes were incubated with horseradish peroxidase-conjugated secondary antibodies (1:5,000; cat no. 7054; Cell Signaling Technology, Inc., Danvers, MA, USA) for $2 \mathrm{~h}$ at room temperature. Enhanced chemiluminescence reagent (Beyotime Institute of Biotechnology) was used to develop the protein and semi-quantified using Bio-Rad Laboratories Quantity One software 3.0 (Bio-Rad Laboratories, Inc., Hercules, CA, USA).
Statistical analysis. Data were represented as the mean \pm standard deviation of three independent experiments. All data were analyzed with one-way analysis of variance followed by Tukey's post-hoc test using SPSS 16.0 (SPSS, Inc., Chicago, IL, USA). $\mathrm{P}<0.05$ was considered to indicate a statistically significant difference.

\section{Results}

Expression of miR-195 in ovarian cancer. To observe the association between the expression of miR-195 and human 

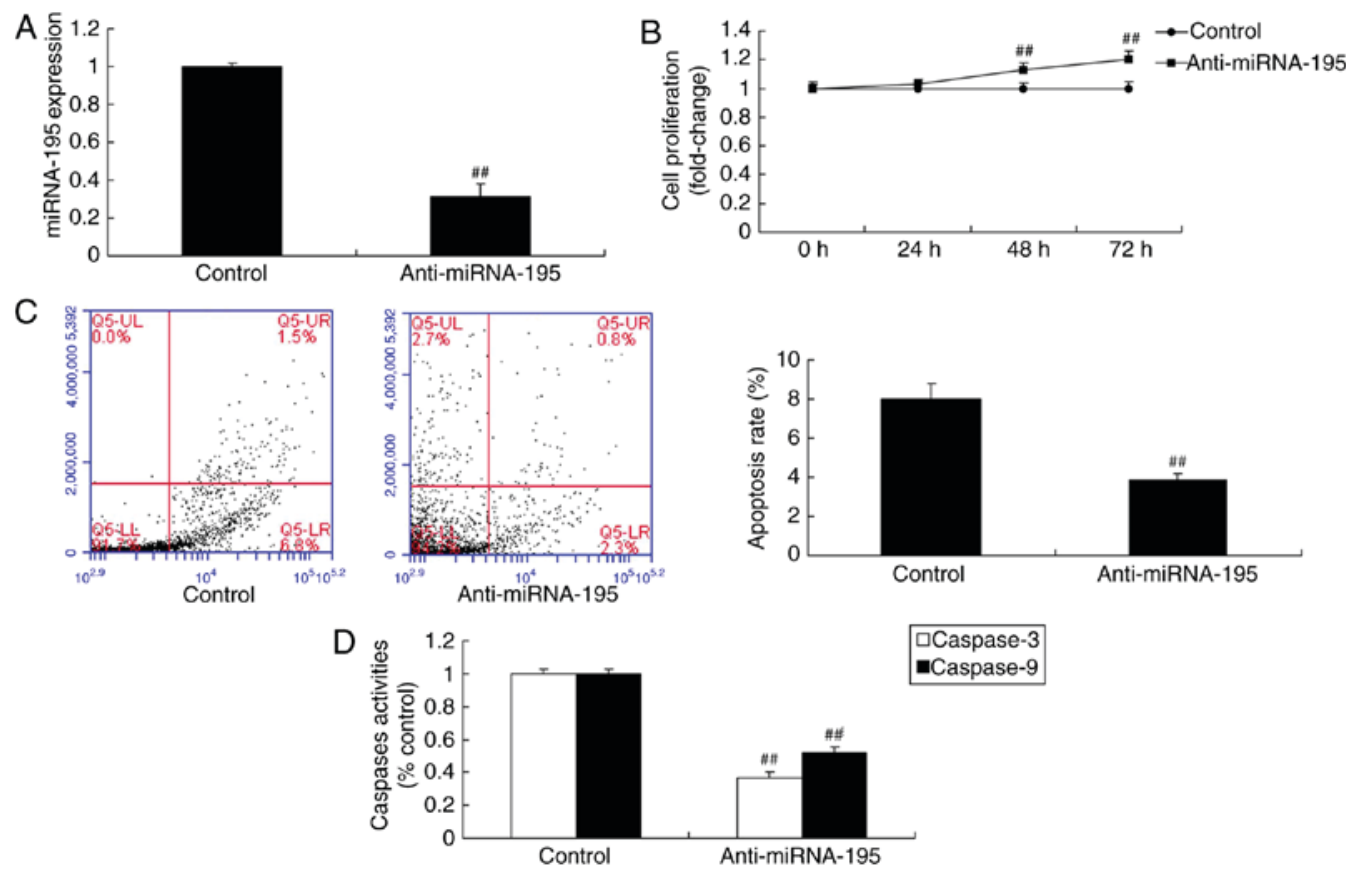

Figure 3. miRNA-195 inhibitor increases cell proliferation and inhibits apoptosis of ovarian cancer cells. (A) Expression of miRNA-195, (B) cell proliferation and (C) apoptosis rate. Q5-UR and Q5-LR were designated as apoptotic cells. (D) Caspase-3/9 activity. ${ }^{\# \#} \mathrm{P}<0.01$ vs. control. Control, negative mimic control group; Anti-miRNA, microRNA inhibitor; UR, upper right; UL, upper left; LR, lower right; LL, lower left.
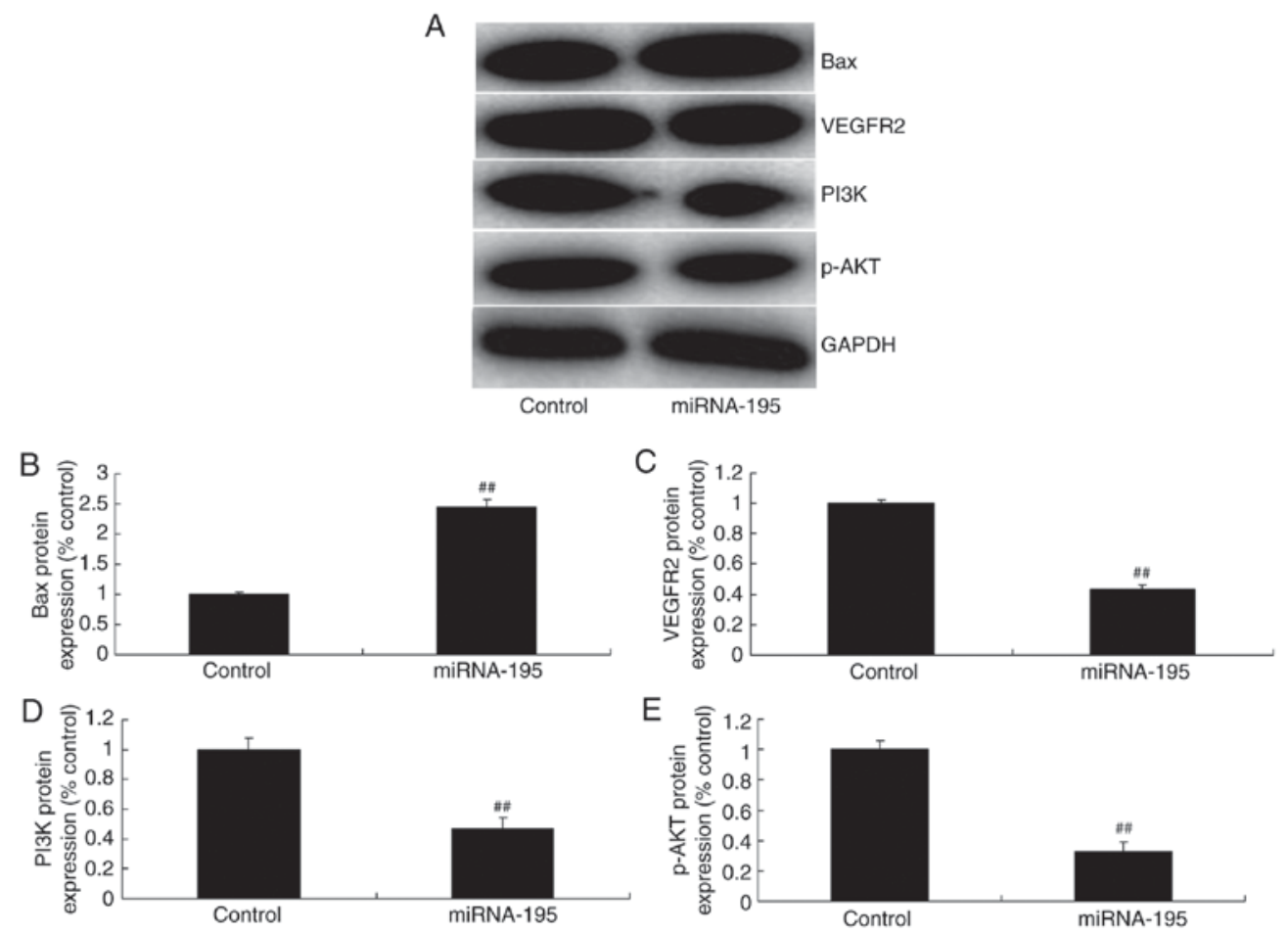

Figure 4. miRNA-195 induces Bax and suppresses VEGFR2 and p-AKT protein expression in ovarian cancer cells. (A) Western blot analysis and densitometry analysis of (B) Bax, (C) VEGFR2, (D) PI3K and (E) p-AKT protein expression, "\# $\mathrm{P}<0.01$ vs. control. Bax, Bcl2 associated $\mathrm{X}$ apoptosis regulator; VEGFR2, vascular endothelial growth factor receptor 2; PI3K, phosphoinositide 3-kinase; p-AKT, phosphorylated AKT; Control, negative mimic control group; miRNA-195, microRNA-195.

ovarian cancer, RT-qPCR was used to measure the expression of miR-195 levels. As presented in Fig. 1, the expression of miR-195 was significantly downregulated in ovarian cancer serum samples, compared with the control normal group $(\mathrm{P}<0.01)$. These data suggest that the expression of miR-195 may be implicated in the pathogenesis of human ovarian cancer.

miR-195 suppresses cell proliferation and induces apoptosis of ovarian cancer cells. To validate the effects of miR-195 on 


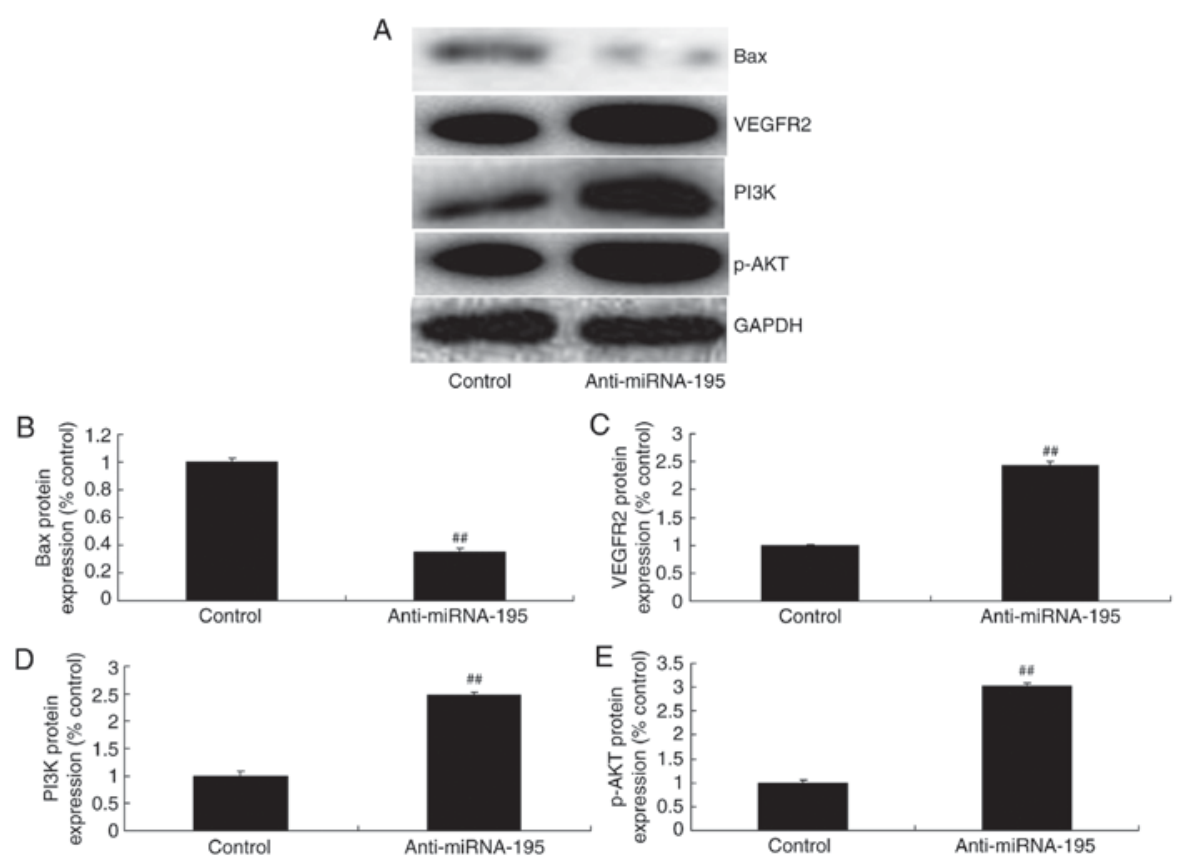

Figure 5. miRNA-195 inhibitor suppresses Bax and induces VEGFR2 and p-AKT protein expression in ovarian cancer cells. (A) Bax, (B) VEGFR2, (C) PI3K and (D) p-AKT protein expression by statistical analysis, and (E) western blotting. ${ }^{\# \#} \mathrm{P}<0.01$ vs. the control group. Bax, Bcl2 associated $\mathrm{X}$ apoptosis regulator; VEGFR2, vascular endothelial growth factor receptor 2; PI3K, phosphoinositide 3-kinase; p-AKT, phosphorylated protein kinase B; Control, negative mimic control group; Anti-miRNA-195, microRNA-195 inhibitor.

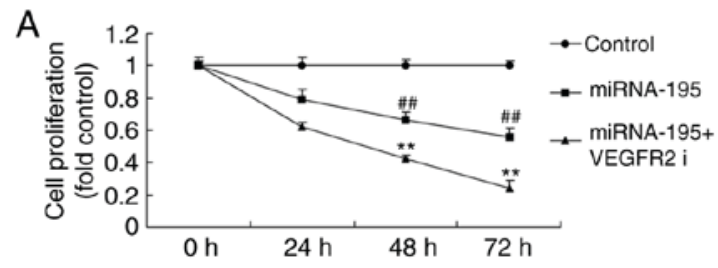

B
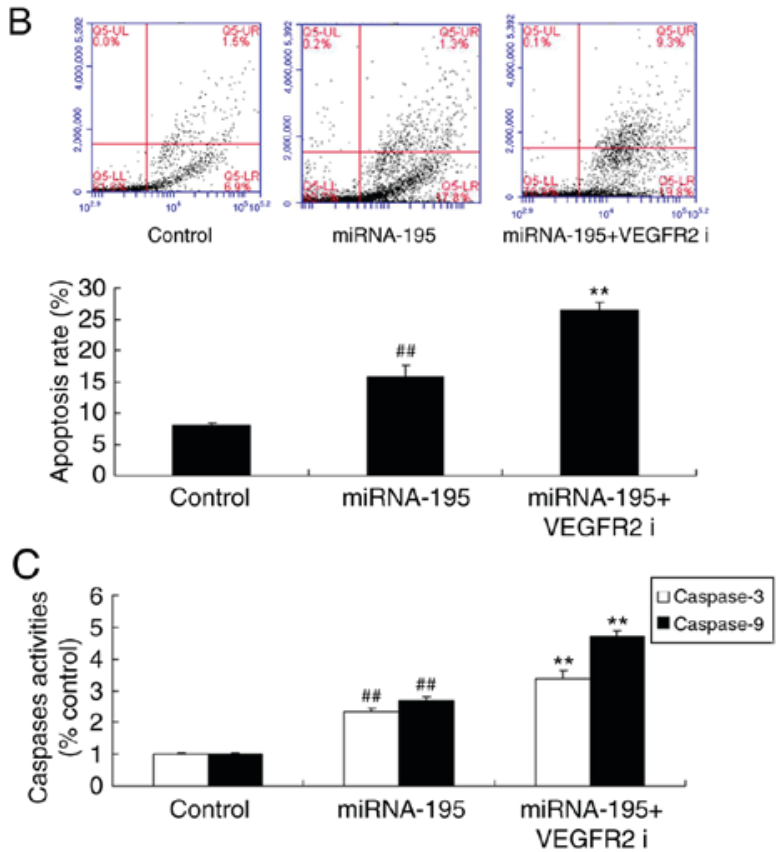

Figure 6. Inhibition of VEGFR2 increases the functional effects of miRNA-195 on apoptosis of ovarian cancer cells. (A) Cell proliferation (B) apoptosis rate and (C) caspase-3/9 activity. ${ }^{\# \#} \mathrm{P}<0.01$ vs. control group, ${ }^{* *} \mathrm{P}<0.01$ vs. microRNA-195 over-expression group. Control, negative mimic control group; miRNA-195, microRNA-195; VEGFR2 i, vascular endothelial growth factor receptor 2 inhibitor vandetanib. the growth and death of OVCAR-3 ovarian cancer cells, an miR-195 mimic was used to significantly increase miR-195 expression ( $\mathrm{P}<0.01$; Fig. 2A). Fig. 2B-D demonstrated that miR-195 suppressed cell proliferation, and induced apoptosis and caspase-3/9 activity in ovarian cancer cells, compared with the control group.

miR-195 inhibitor increases cell proliferation and inhibits apoptosis of ovarian cancer cells. An miR-195 inhibitor mimic was used to significantly reduce miR-195 expression, and the miR-195 inhibitor significantly increased cell proliferation and inhibited apoptosis of ovarian cancer cells $(\mathrm{P}<0.01$; Fig. 3$)$.

miR-195 induces Bax and suppresses VEGFR2 and p-AKT protein expression in ovarian cancer cells. The effect of miR-195 on apoptosis of ovarian cancer cells, was observed and the VEGFR2 and PI3K/AKT signaling pathway was selected for investigation. As demonstrated in Fig. 4, miR-195 significantly induced Bax and suppressed VEGFR2, PI3K and p-AKT expression in ovarian cancer cells, compared with the control group ( $\mathrm{P}<0.01$; Fig. 4).

miR-195 inhibitor suppresses Bax and induces VEGFR2 and $p$-AKT protein expression in ovarian cancer cells. The anti-miR-195 significantly suppressed Bax expression, and induced VEGFR2, PI3K and p-AKT expression in ovarian cancer cells, compared with the control group ( $\mathrm{P}<0.01$; Fig. 5). These results demonstrate that the role of VEGFR2 and PI3K/AKT signaling pathways in miR-195 induced apoptosis regulation requires further investigation.

Inhibition of VEGFR2 increases the functional effects of miR-195 on apoptosis of ovarian cancer cells. To investigate 


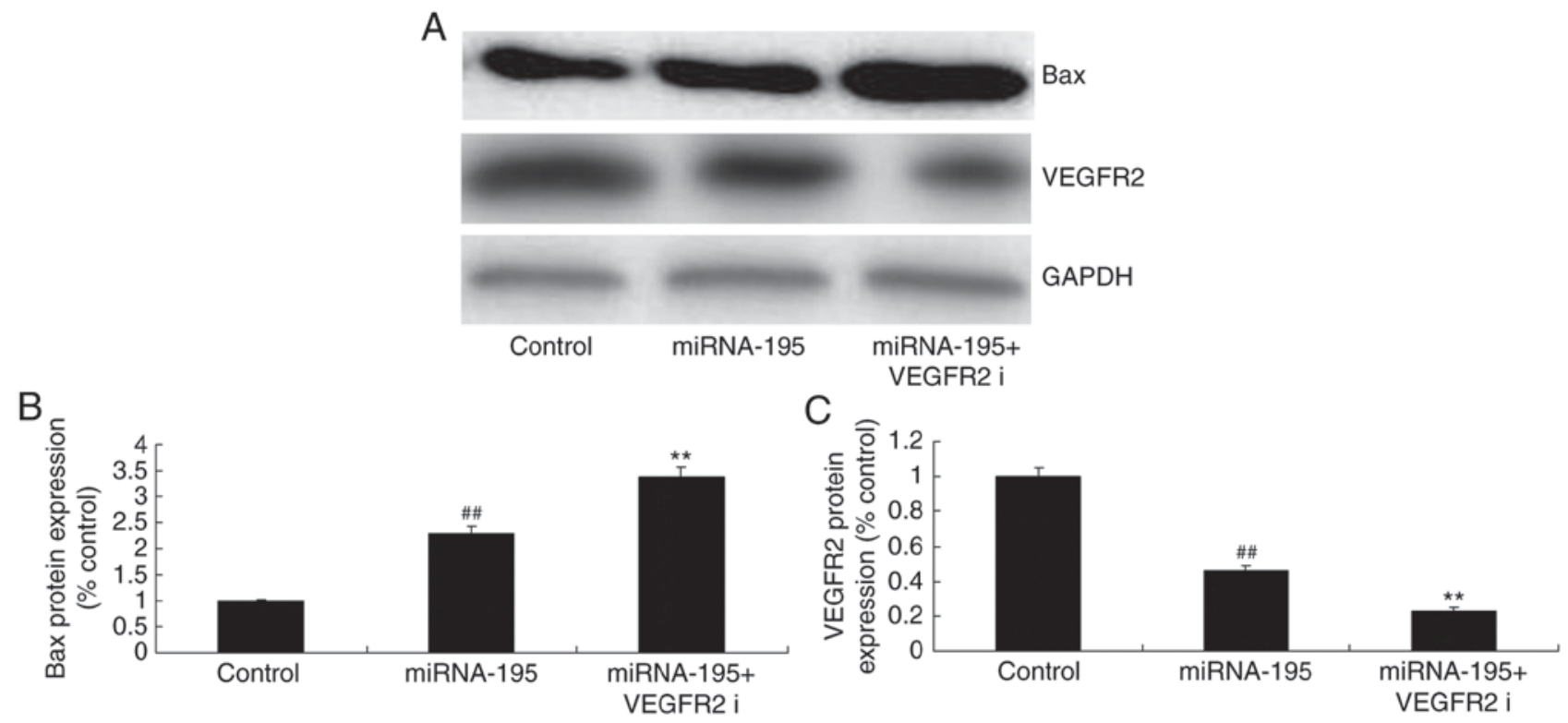

Figure 7. Inhibition of VEGFR2 increases the functional effects of microRNA-195 on Bax and induced VEGFR2 of ovarian cancer cells. (A) Western blotting and densitometry was used to analyze (B) Bax and (C) VEGFR2 protein expression. ${ }^{\# \#} \mathrm{P}<0.01$ vs. the control group, ${ }^{* *} \mathrm{P}<0.01$ vs. microRNA-195 over-expression group. Bax, Bcl2 associated X apoptosis regulator; VEGFR2, vascular endothelial growth factor receptor 2; Control, negative mimic control group; miRNA-195, microRNA-195; VEGFR2 i, VEGFR2 inhibitor vandetanib.

the role of miR195 in regulating VEGFR2 in ovarian cancer cells, a VEGFR2 inhibitor, vandetanib $(10 \mathrm{nM})$ was added to cells following transfection with miR-195 mimics. Vandetanib significantly increased the functional effects of miR-195 on the inhibition of cell proliferation, and the promotion apoptosis and caspase-3/9 activity of ovarian cancer cells, compared with miR-195 group (Fig. 6). Furthermore, the inhibition of VEGFR2 significantly suppressed VEGFR2 and increased Bax protein expression (P<0.01; Fig. 7).

Inhibition of AKT increases the functional effects of miR-195 on apoptosis of ovarian cancer cells. The role of AKT on the functional effects of miR-195 on apoptosis of ovarian cancer cells was investigated. An AKT inhibitor LY294002 $(100 \mathrm{nM})$, increased the functional effects of miR-195 on apoptosis of ovarian cancer cells (Fig. 8), and increased Bax, and suppressed PI3K and p-AKT expression, compared with the miR-195 group (Fig. 9).

\section{Discussion}

Ovarian cancer is a common malignancy of the female genital tract. Epithelial ovarian cancer is the most common histopathological type of ovarian cancer (1). The mortality rate for ovarian cancer is the highest of all gynecological malignancies (16). Ovarian cancer has silent onset and $\sim 2 / 3$ patients are in the advanced stage at the onset of symptoms (16). In the current study, the expression of miR-195 was downregulated in ovarian cancer serum samples, compared with the normal control group.

miRs can directly regulate the genesis and development of ovarian cancer (17). The known mechanisms include miR gene deletion and epigenetic modification (17). In addition, abnormal expression of key enzymes involved in miR
A

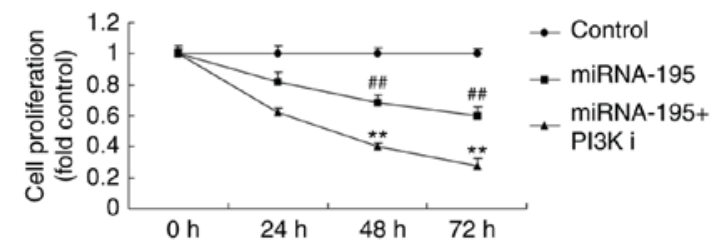

B

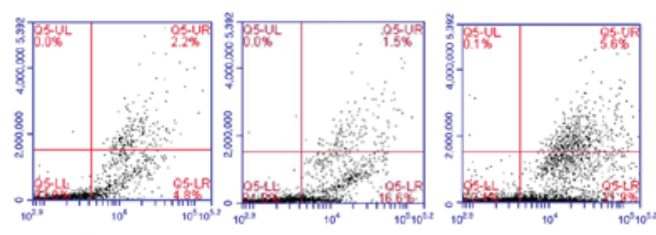

Control
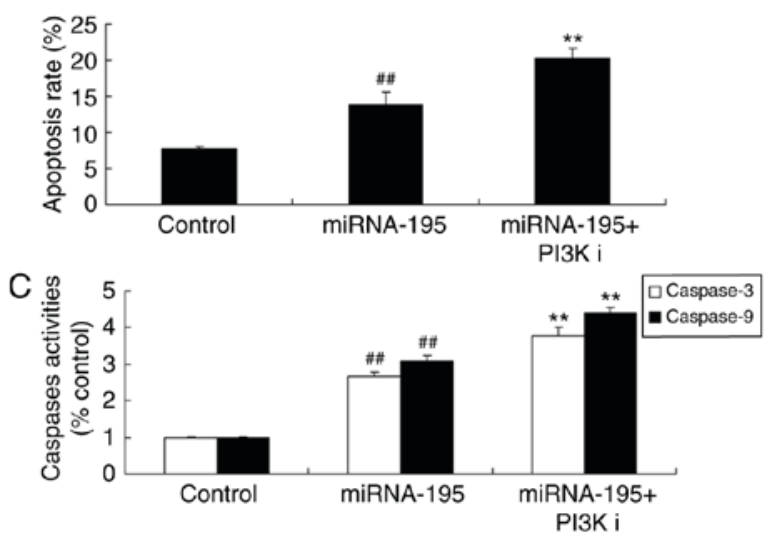

Figure 8. Inhibition of PI3K increases the functional effects of miRNA-195 on apoptosis of ovarian cancer cells. (A) Cell proliferation, (B) apoptosis rate and (C) caspase-3/9 activity. ${ }^{\# \#} \mathrm{P}<0.01$ vs. the control group, ${ }^{* *} \mathrm{P}<0.01$ vs. the miRNA-195. PI3K, phosphoinositide 3-kinase; Control, negative mimic control group; miRNA-195, microRNA-195; PI3K i, PI3K inhibitor LY294002.

synthesis is also involved in the development of ovarian cancer. Further studies on the differentially expressed miRs 
A
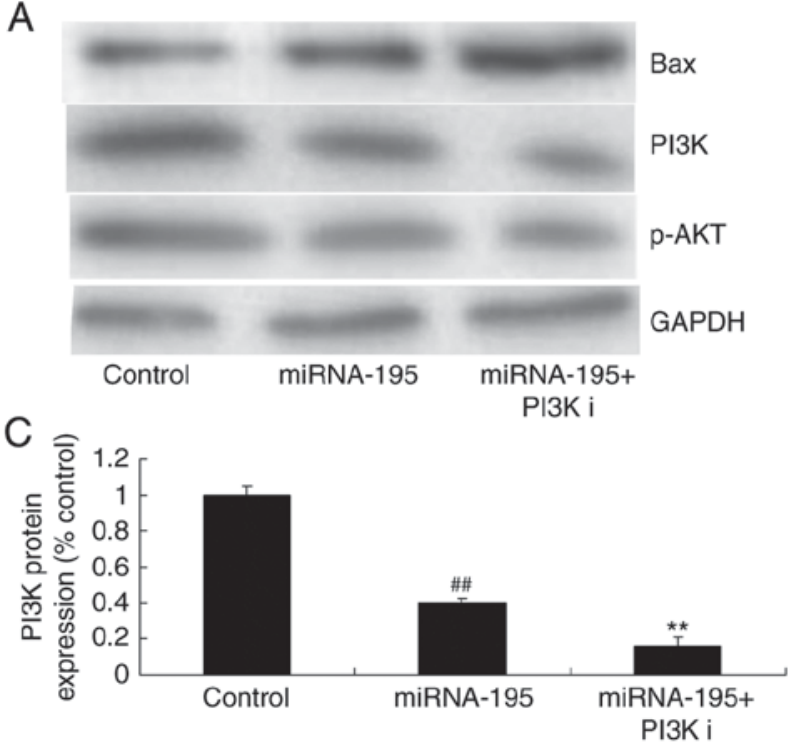

B
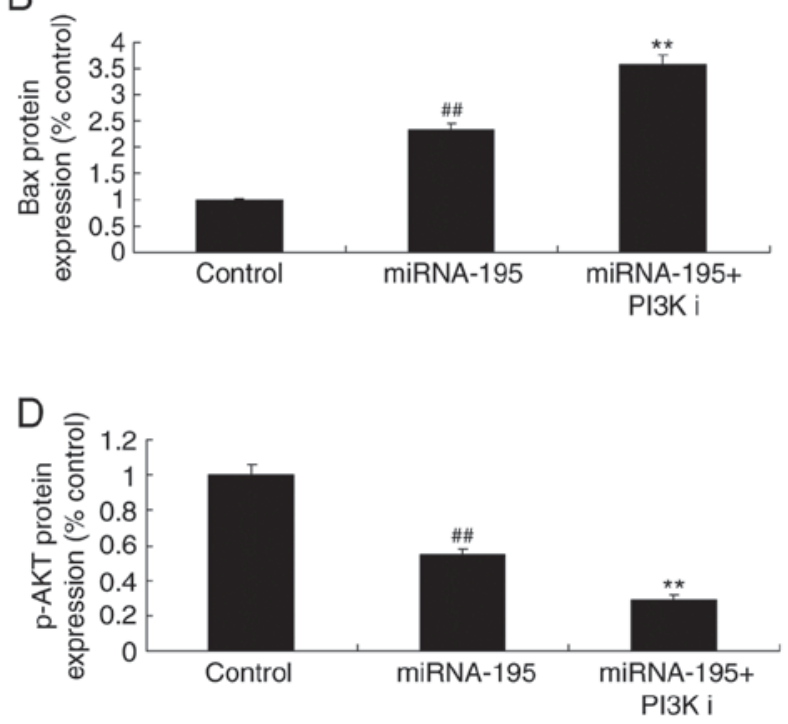

Figure 9. Inhibition of PI3K increased the functional effects of miRNA-195 on Bax and induced VEGFR2 in ovarian cancer cells. (A) Western blotting and densitometry was used to determine protein expression of (B) Bax, (C) PI3K and (D) p-AKT. ${ }^{\# \#} \mathrm{P}<0.01$ vs. the control group, ${ }^{* *} \mathrm{P}<0.01$ vs. the miRNA-195. Bax, $\mathrm{Bcl} 2$ associated $\mathrm{X}$ apoptosis regulator; PI3K, phosphoinositide 3-kinase; p-AKT, phosphorylated protein kinase B; Control, negative mimic control group; miRNA-195, microRNA-195; PI3K i, PI3K inhibitor LY294002.

in tissue and serum of patients with ovarian cancer should be performed. This is of great important for the diagnosis and treatment of ovarian cancer (18). The results of the present study demonstrated that miR-195 suppressed cell proliferation and induced apoptosis of ovarian cancer cells. Only the OVCAR-3 cell line was used, which is a deficiency in the present study and other cell lines will be used in future studies.

VEGFA in the VEGF protein family is crucial in angiogenesis (19). The biological functions of VEGF are achieved through binding with receptors on the target cell surface (19). VEGFR2 is the major receptor through which VEGF family proteins exert their biological functions (8). There are autocrine or paracrine loops of VEGFA and VEGFR2 in tumor cells and vascular endothelial cells, which stimulate angiogenesis (17). Notably, the present study demonstrated that miR-195 suppressed VEGFR2 protein expression in ovarian cancer cells. Sun et al (14) demonstrated that miR-195 suppresses angiogenesis and metastasis of hepatocellular carcinoma through VEGF expression. Wang et al (20) reported that miR-195-5p inhibits tumorigenesis of gastric cancer.

AKT is the downstream target of PI3K. The phosphorylated form of AKT is its active form. The activated phosphorylated AKT detaches from the cell membrane (12). Subsequently, it moves through the cytoplasm or to the cell nucleus. It can therefore activate or inhibit downstream protein molecules (21). The downstream protein molecules include mammalian target of rapamycin, Bad, caspase-9, tuberin, glycogen synthase kinase-3 $\beta$ and the forkhead transcription factor family. Additionally, it can also mediate cell apoptosis, proliferation and angiogenesis (22). In the present study, it was demonstrated that miR-195 suppressed the p-AKT level in ovarian cancer cell. Sun et al (14) indicated that miR-195 suppressed cell growth in renal cell carcinoma via PI3K/AKT signaling pathways. However, only p-AKT expression was measured in the present study, not total AKT expression. Thus, the exact effect of miR-195-5p on AKT cannot be determined and requires further study.

In summary, miR-195 suppressed cell proliferation and induced the apoptosis of ovarian cancer cells through suppression of VEGFR2 and AKT signaling pathway proteins. These findings indicate that miR-195 may be a novel noninvasive biomarker, which would provide considerable diagnostic value in screening for ovarian cancer.

\section{Acknowledgements}

Not applicable.

\section{Funding}

Not applicable.

\section{Availability of data and materials}

The analyzed data sets generated during the study are available from the corresponding author on reasonable request.

\section{Authors' contributions}

JC designed the experiment, performed the experiment, analyzed the data and wrote the manuscript.

\section{Ethics approval and consent to participate}

The study protocol was approved by the ethics committee of the Third Affiliated Hospital of Qiqihar Medical College (Qiqihar, China). Written informed consent was provided by all participants. 


\section{Consent for publication}

Not applicable.

\section{Competing interests}

The author declares that they have no competing interests.

\section{References}

1. Li X, Du N, Zhang Q, Li J, Chen X, Liu X, Hu Y, Qin W, Shen N, $\mathrm{Xu}$ C, et al: MicroRNA-30d regulates cardiomyocyte pyroptosis by directly targeting foxo3a in diabetic cardiomyopathy. Cell Death Dis 5: e1479, 2014.

2. Wu C, Jin B, Chen L, Zhuo D, Zhang Z, Gong K and Mao Z: MiR-30d induces apoptosis and is regulated by the Akt/FOXO pathway in renal cell carcinoma. Cell Signal 25: 1212-1221, 2013.

3. Xin Q, Li J, Dang J, Bian X, Shan S, Yuan J, Qian Y, Liu Z, Liu G, Yuan Q, et al: miR-155 deficiency ameliorates autoimmune inflammation of systemic lupus erythematosus by targeting S1pr1 in Faslpr/lpr mice. J Immunol 194: 5437-5445, 2015.

4. Mi L, Chen Y, Zheng X, Li Y, Zhang Q, Mo D and Yang G: MicroRNA-139-5p suppresses 3T3-L1 preadipocyte differentiation through notch and IRS1/PI3K/Akt insulin signaling pathways. J Cell Biochem 116: 1195-1204, 2015.

5. Krishnan K, Steptoe AL, Martin HC, Pattabiraman DR, Nones K, Waddell N, Mariasegaram M, Simpson PT, Lakhani SR, Vlassov A, et al: miR-139-5p is a regulator of metastatic pathways in breast cancer. RNA 19: 1767-1780, 2013.

6. Maoa R, Zou F, Yang L, Lin S, Li Y, Ma M, Yin P, Liang X and Liu J: The loss of MiR-139-5p promotes colitis-associated tumorigenesis by mediating PI3K/AKT/Wnt signaling. Int J Biochem Cell Biol 69: 153-161, 2015.

7. Zhang Y, Zhang D, Wang F, Xu D, Guo Y and Cui W: Serum miRNAs panel (miR-16-2*, miR-195, miR-2861, miR-497) as novel non-invasive biomarkers for detection of cervical cancer. Sci Rep 5: 17942, 2015.

8. Alevizos I and Illei GG: MicroRNAs in Sjögren's syndrome as a prototypic autoimmune disease. Autoimmun Rev 9: 618-621, 2010.

9. Mameli G, Arru G, Caggiu E, Niegowska M, Leoni S, Madeddu G, Babudieri S, Sechi GP and Sechi LA: Natalizumab therapy modulates miR-155, miR-26a and proinflammatory cytokine expression in MS patients. PLoS One 11: e0157153, 2016.

10. Liu J, Xiao X, Shen Y, Chen L, Xu C, Zhao H, Wu Y, Zhang Q, Zhong J, Tang Z, et al: MicroRNA-32 promotes calcification in vascular smooth muscle cells: Implications as a novel marker for coronary artery calcification. PLoS One 12: e0174138, 2017.
11. Li M and Zhang J: Circulating MicroRNAs: Potential and emerging biomarkers for diagnosis of cardiovascular and cerebrovascular diseases. Biomed Res Int 2015: 730535, 2015.

12. Ke ZP, Xu P, Shi Y and Gao AM: MicroRNA-93 inhibits ischemia-reperfusion induced cardiomyocyte apoptosis by targeting PTEN. Oncotarget 7: 28796-28805, 2016.

13. Schulte C, Karakas M and Zeller T: microRNAs in cardiovascular disease-clinical application. Clin Chem Lab Med 55: 687-704, 2017.

14. Sun P, Wang L, Lu Y, Liu Y, Li L, Yin L, Zhang C, Zhao W, Shen B and Xu W: MicroRNA-195 targets VEGFR2 and has a tumor suppressive role in ACHN cells via PI3K/Akt and Raf/MEK/ERK signaling pathways. Int J Oncol 49: 1155-1163, 2016.

15. Livak KJ and Schmittgen TD: Analysis of relative gene expression data using real-time quantitative PCR and the 2(-Delta Delta $\mathrm{C}(\mathrm{T}))$ method. Methods 25: 402-408, 2001.

16. Chen JQ, Papp G, Póliska S, Szabó K, Tarr T, Bálint BL, Szodoray $\mathrm{P}$ and Zeher M: MicroRNA expression profiles identify disease-specific alterations in systemic lupus erythematosus and primary Sjögren's syndrome. PLoS One 12: e0174585, 2017.

17. Jimenez SA and Piera-Velazquez S: Potential role of human-specific genes, human-specific microRNAs and human-specific non-coding regulatory RNAs in the pathogenesis of systemic sclerosis and Sjogren's syndrome. Autoimmun Rev 12: 1046-1051, 2013.

18. Williams AE, Choi K, Chan AL, Lee YJ, Reeves WH, Bubb MR, Stewart CM and Cha S: Sjögren's syndrome-associated microRNAs in CD14(+) monocytes unveils targeted TGF $\beta$ signaling. Arthritis Res Ther 18: 95, 2016.

19. Tandon M, Gallo A, Jang SI, Illei GG and Alevizos I: Deep sequencing of short RNAs reveals novel microRNAs in minor salivary glands of patients with Sjögren's syndrome. Oral Dis 18: 127-131, 2012.

20. Wang J, Li L, Jiang M and Li Y: MicroRNA-195 inhibits human gastric cancer by directly targeting basic fibroblast growth factor. Clin Transl Oncol 19: 1320-1328, 2017.

21. Ustun N, Aras M, Ozgur T, Bayraktar HS, Sefil F, Ozden R and Yagiz AE: Thymoquinone attenuates trauma induced spinal cord damage in an animal model. Ulus Travma Acil Cerrahi Derg 20: 328-332, 2014.

22. Ohta K, Hoshino H, Wang J, Ono S, Iida Y, Hata K, Huang SK, Colquhoun S and Hoon DS: MicroRNA-93 activates c-Met/PI3K/Akt pathway activity in hepatocellular carcinoma by directly inhibiting PTEN and CDKN1A. Oncotarget 6: 3211-3224, 2015. 